\title{
Research on Landscape Architecture Design Based on Corporate Social Responsibilities
}

\author{
Xinying Zhang ${ }^{1, *}$, Wenjie Chen ${ }^{2}$ \\ ${ }^{1}$ College of Landscape Architecture, Sichuan Agricultural University, Chengdu 611130, P.R. China \\ ${ }^{2}$ School of Economics and Management, Chongqing University of Posts and Telecommunications, \\ Chongqing 400065, P.R. China
}

\begin{abstract}
As the natural environment becomes growingly deteriorated, environmental protection becomes a global common concern. Landscape architecture (LA) is concerned about the relationship among humans, the built, and natural environments, so it is of special significance to study corporate social responsibilities (CSR) of LA firms. This paper studied CSR of LA firms from the perspective of landscape architects, i.e., this paper explored LA design based on corporate social responsibilities. CSR of LA firms was classified into economic responsibilities and green responsibilities, so this paper studied LA design based on economic responsibilities and green responsibilities respectively. This paper contributes to the existing study of LA design by innovatively adding corporate social responsibilities into the LA design philosophies.
\end{abstract}

\section{Introduction}

With the rapid economic development, eco-environmental problems such as resource shortage, global warming, urban heat island effect, and ecological pollution occur frequently. Under this background, environmental protection becomes a global common concern, and a good ecological environment becomes the basic conditions and common demands for the economic and social development of all countries, so green development and protection of the eco-environment become the common objective for all countries. Landscape architecture (hereinafter abbreviated as LA) deals with the relationship among humans, the built and natural environments, so it plays an especially important role in protecting the eco-environment and facilitating green development in ways such as improving human living spaces, saving energies, enriching biodiversity, and preserving historic and cultural relics. Due to the inherently distinctive nature of LA, LA firms shoulder greater social responsibilities than any other firms to protect the ecological environment. As employees, landscape architects are assumed to take active initiative to help the firm they serve to perform its social responsibilities through the LA design.

\section{Literature review}

The concept of green responsibility evolves from corporate social responsibility. Since the concept of corporate social responsibility (hereinafter abbreviated as CSR) was first proposed by Oliver Sheldon in 1923, numerous scholars have done extensive research on CSR.
Different scholars have made different descriptions of CSR from different perspectives. Carroll (1991) proposed a classic four-level pyramid model of CSR, as shown in Fig.1, indicating that economic responsibilities at the bottom of the pyramid are the required basic social responsibility for all firms.

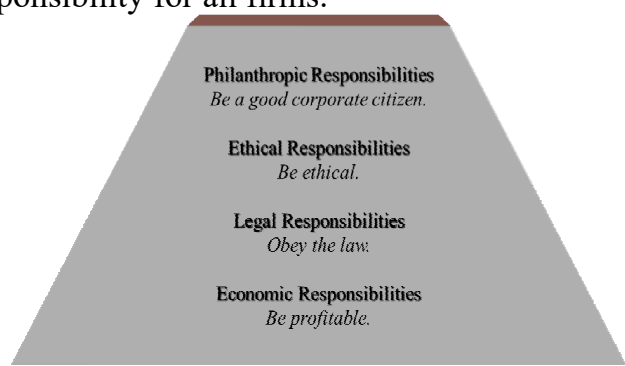

Fig.1. The Pyramid of Corporate Social Responsibility

Source: A. B. Carroll. The Pyramid of Corporate Social Responsibility: Toward the Moral Management of Organizational Stakeholders [J]. Business Horizons, July/August: 39-48 (1991).

CSR means that a firm should not only act in the best interests of its shareholders but also be responsible for all of its stakeholders, including employees, society, and environment. Accordingly, the concept of green CSR or green responsibility was proposed. A firm's green responsibility or green CSR refers to the firms' social responsibility in environmental protection and resource utilization. Specifically speaking, green responsibility involves that a firm should strive to avoid resource waste and minimize its destruction to the environment during its operation process, i.e., be responsible for the eco-environmental protection. To maintain harmonious co-existence among humans, nature, and society and facilitate the sustainable development of humans, nature,

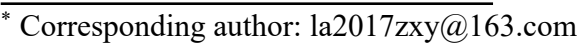


and society, firms are assumed to undertake green responsibilities. Green responsibilities are one domain of CSR, layered upon economic responsibilities.

Only two papers in Chinese are available on http://www.cnki.net that are concerned about the corporate social responsibilities of LA firms. Zhang et al. (2010) started with the corporate social responsibility (CSR), described its basic concept that contains various corporate activities, and discussed the way Japanese LA firms perform their corporate social responsibilities with three related examples of Japanese landscape. Wu (2009) suggested that LA firms should undertake the social responsibility of being a green firm and argued that the social responsibility of LA firms include 6 dimensions: commercial civilization, science and technology innovation, corporate culture, green practice, client support, and co-construction with other stakeholders.

LA firms plays a particularly important role in reducing resource waste and minimizing destruction to the environment, so it is of special significance to study CSR of LA firms. Without a doubt, LA firms should be environmentally responsible. As employees of a LA firm, landscape architects should also be environmentally responsible through their LA design works. Thus, this paper attempts to analyse CSR of LA firms from the perspective of landscape architects. In other words, this paper intends to explore LA design based on corporate social responsibilities.

\section{LA design based on the economic responsibilities}

A firm's primary responsibility is to make profits, i.e., the economic responsibility is the basic responsibility upon which all other responsibilities are based (Carroll,1991). Friedman (1970) also maintained that the social responsibility of business is to increase its profits. No firm can survive or develop on the market without profits over the time. Thus, landscape architects should endeavor to meet the economic responsibilities of both the firm and the society.

\subsection{The economic responsibilities of LA design for the firm}

To help the firm they serve to perform its economic responsibilities, i.e., maximize its profits, landscape architects are supposed to think strategically and act economically. They should make efforts to help the firm to make profits through their design works, i.e., to produce LA design products which enable the firm to obtain greater corporate performance with less costs. Put it another way, LA design products are supposed to serve the competitive strategy the firm has formulated. As for the firm adopting a cost leadership strategy, landscape architects are assumed to produce competitive LA products with relatively low costs, e.g., by adopting less complicated design processes or selecting low-cost building materials. In terms of the firm with a differentiation strategy, landscape architects should make efforts to produce distinctive LA products with various meaningful and aesthetic functions, say, by using unique design processes and selecting diversified building materials. In a word, it is one of the social responsibilities of landscape architects to help the firm in which they work to meet its economic responsibilities.

\subsection{The economic responsibilities of LA design for the society}

As environmentally responsible employees, landscape architects are also assumed to shoulder the social responsibility for the society. They should try to produce LA design products capable of generating social benefits and improving social well-being. Landscape architects are supposed to help to relieve the tensions between humans and the natural environments with their design works. For example, landscape architects may in some way ease the contradictions between LA and human demands for water by designing water-saving ecological parks. Besides, landscape architects should be conscious of designing various works that can protect and improve the urban environments which in turn facilitate the economic development of the surrounding areas. Put it another way, landscape architects are assumed to shoulder the responsibility of boosting the economic and social development or improve the total social well-being of the local area with their design works.

In the past decades, many local Chinese governments have paid increased attentions to LA design with an attempt to attract tourists and thus foster the development of the local tourism industry. Numerous successful cases of LA design are available throughout China, among which Chongqing may probably be recognized as the most typical one. Since 1990s, the Chongqing government have invested much in LA design according to the distinctive topographical and geomorphological conditions featured by a combination of rivers and hills with various altitudes. Chongqing is currently well-known for its unique nightscape lighting due to the local government's continuous efforts in LA design in the past years. Millions of tourists rush to Chongqing from different parts of China and even the world for sightseeing, bringing significant economic benefits to Chongqing. Landscape architects have undoubtedly made a great contribution to the economic and social development of Chongqing.

\section{LA design based on the green responsibilities}

Economic responsibilities are the basic responsibility for all firms. As for LA firms, the most important responsibility is the green responsibilities. Thus, the following sections focuses on the way LA firms perform their green responsibilities from the perspective of landscape architects. The work of landscape architects is always involved with the three types of relationship: relationship between humans and the built environment, relationship between humans and the natural environments, and relationship between the built and natural environments. Thus, the following sections 
attempt to explore the way in which landscape architects deal with the three types of relationship based on the green responsibilities.

\subsection{Trying to achieve an effective unification of meaningful and aesthetic LA with human demands for better living spaces}

LA design based on environmental responsibility focuses on the sustainable development of the ecological environment, but it never overlooks the social nature and aesthetic characteristics of LA. To coordinate the relationship between humans and the built environment, landscape architects should endeavor to design LA that is both meaningful and aesthetic so as to provide pleasing relaxation, leisure, and recreation places for humans. For example, when landscape architects are designing water landscape like fountains, they should first consider if the water landscape can bring an extraordinary visual effect for viewers, and meanwhile they should also value if the water landscape can be reached by viewers at short range. For another example, when landscape architects are designing park roads, they should consider both the roads' function to evacuate sight viewers and the sight viewers' aesthetic demands. To do so, landscape architects may design an over 100 meters long road covered with cobblestone that is wide enough to allow at least two pedestrians to walk simultaneously in parallel. In this way, sight viewers can experience a soothing feeling in their feet soles touching cobblestone and meanwhile watch the enjoyable green scenery along the $\operatorname{road}(\mathrm{Hu}, 2020)$.

\subsection{Trying to realize an organic combination of human demands for a better living environment with respect for the natural environment}

With the economic and social development, people have greater demands for a better life quality than ever before, including a better living environment. To achieve the purpose of living in a better environment, people have been used to overexploitation of the natural resources, resulting in destruction and deterioration of the present natural environment. LA design is involved with the reshaping and optimization of the natural environment, so it may inevitably do some destruction to the existing natural environment. Thus, landscape architects should try every means to minimize the destruction of their design works to the natural environment and meanwhile meet humans' demands for pleasing living spaces. To achieve the above dual objective, landscape architects may conduct LA design according to the following steps (Wu, 2019).

(1) Before the LA design, landscape architects should make an in-depth investigation of the existing natural conditions in the landscape, and then propose a design scheme proper for the local conditions. Information necessary for the investigation usually include climate features, topographical and geomorphological conditions, hydrology, municipal facilities, traffic conditions, and plants.
(2) In terms of the selection of plants, local plants are preferred. If landscape architects find it necessary to introduce alien species of plants from other places, they must select those species adaptable to the local conditions.

(3) During the construction process of LA, landscape architects should stick to the belief of minimal destruction to or minimal reshaping of the natural environment in order to demonstrate the LA characteristics based on the protection of the local environment.

\subsection{Trying to make a good coordination of the construction of modern LA with the preservation of historic and cultural relics}

Areas vary in geography, history, culture, and economic development. Each area has its own geographical, historical, and cultural characteristics and is at a certain economic development stage. Before the LA design, landscape architects need to have a better understanding of the unique historical and cultural characteristics and the economic development level of the aera involved. They should incorporate the development characteristics into the historical tradition of the local area. Their LA design should show respect for the history and meanwhile be oriented toward the future. In other words, LA design should be intended to preserve historic and cultural sites whenever possible for the purpose of preventing the natural environment form being destroyed. Besides, LA design should also be aimed to demonstrate the economic and technological development characteristics of the local area to enable people to feel the change of time.

East Chendu Memory is a successful LA design case combing the construction of modern LA with the preservation of historic and cultural relics. East Chendu Memory was built at the old site of a former state-run electronic tube factory founded in the 1950s. At the old site, some of the factory areas with typical industrial characteristics were preserved as an industrial civilization relic, and the remaining areas were reconstructed into a cultural and recreational base. In terms of architectural style, East Chendu Memory combined the industrial tradition with the music fashion. Here, people can feel the fascination of an industrial relic, experience the evolution of the local city, and recall the past happy memories ${ }^{1}$.

\section{Conclusions}

We live in an era when the natural environment becomes increasingly deteriorated. Any individual and organization has the social responsibility to protect the natural environment from being destroyed in order to achieve a sustainable harmonious development between humans and the nature. LA is concerned about the

\footnotetext{
1 Translation of excerpts from the introduction to East Chendu Memory at https://baike.baidu.com/item/东郊记忆 /6249828?fr=aladdin
} 
relationship among humans, the built, and natural environments. Thus, it is of special significance to study CSR of LA firms. This paper has studied CSR of LA firms from the perspective of LA design. CSR of LA firms was classified into economic responsibilities and green responsibilities. Accordingly, this paper has discussed in detail LA design based on economic responsibilities and green responsibilities respectively.

\section{Acknowledgment}

We would like to thank the anonymous reviewers for their valuable suggestions. Financial support from Chongqing Social Science Found (Project\#2018YBGL079) is also acknowledged.

\section{References}

1. A. B. Carroll. The Pyramid of Corporate Social Responsibility: Toward the Moral Management of Organizational Stakeholders [J]. Business Horizons, July/August: 39-48 (1991)

2. A. Zhang, Y. 1. Zhang, M.L. Kong, J.H. Zhang. Corporate Social Responsibility (CSR) and Landscape architecture-In Order to Achieve Low-carbon Society [J]. Chinese Landscape Architecture, 6:31-34 (2010) (in Chinese)

3. M. Friedman. The Social Responsibility of Business is to Increase Its Profits [N]. New York Times, September 13: 122-126 (1970)

4. G. C. Wu. Green Landscape Enterprises Should Take on Social Responsibilities [J]. Chinese Landscape Architecture, 9:78-79 (2009) (in Chinese)

5. X.T. Wu. Analysis of the Application of Landscape Architecture Design to Environmental Protection [J]. Intelligent City, 19:185-186(2019) (in Chinese)

6. C. R. Hu. Discussion of the Application of Humanized Design Belief in Landscape Architecture Design [J]. Housing and Real Estate, 18:54 (2020) (in Chinese) 